\title{
Ocular involvement in coronavirus disease 2019 (COVID-19): a clinical and molecular analysis
}

\author{
Asaf Shemer • Adi Einan-Lifshitz • Amir Itah • Biana Dubinsky-Pertzov • \\ Eran Pras • Idan Hecht $(\mathbb{D}$
}

Received: 3 June 2020/Accepted: 5 September 2020/Published online: 14 September 2020

(C) Springer Nature B.V. 2020

\begin{abstract}
Purpose Coronavirus disease 2019 (COVID-19) caused a global pandemic with millions infected worldwide. Little is known on the ocular involvement associated with the disease. The aim of this study was to assess the clinical and molecular ocular involvement among patients with confirmed COVID-19 admitted to a tertiary care facility.

Methods Consecutive patients admitted to the COVID-19 Ward of the Shamir Medical Center in Israel during March and April, 2020 were included. The control group included patients negative for COVID-19 admitted during a similar period to a different ward. Patients were examined by trained Ophthalmologists. SARS-CoV-2 conjunctival swab samples were obtained.
\end{abstract}

Electronic supplementary material The online version of this article (https://doi.org/10.1007/s10792-020-01592-1) contains supplementary material, which is available to authorized users.

A. Shemer · A. Einan-Lifshitz · A. Itah ·

B. Dubinsky-Pertzov · E. Pras · I. Hecht ( $\square)$

Department of Ophthalmology, Shamir Medical Center

(Formerly Assaf-Harofeh), Tzrifin, Israel

e-mail: Idan.Hecht@gmail.com

A. Shemer - A. Einan-Lifshitz - B. Dubinsky-Pertzov ·

E. Pras · I. Hecht

Sackler Faculty of Medicine, Tel Aviv University,

Tel Aviv, Israel
Results Included were 48 patients, 16 with confirmed COVID-19 and 32 controls. Median patient age was 68.5 (interquartile range: 31.5 , mean: $63 \pm 21$ ) years and $48 \%$ were male. Active conjunctival injection was present in three patients (19\%) with COVID19 , compared to none in the controls $(p=0.034)$. Patients with COVID-19 were more likely to complain of foreign body sensation $(31.3 \%$ vs $3.1 \%, p=0.005)$ and redness of the eye (25\% vs $0 \%, p=0.003)$. Conjunctival injection was associated with loss of smell and taste $(75 \%$ vs $7.7 \%, p=0.018)$. Viral conjunctival swab tests all showed negative results for all three viral genes tested (E, N, and RdRp).

Conclusions Among patients admitted to a tertiary referral center with confirmed COVID-19, active conjunctival injection was noted in one out of five cases, and was associated with loss of smell and taste. Conjunctival swabs for viral RNA were negative in patients with and without ocular involvement.

\footnotetext{
A. Itah

Faculty of Medicine, The Hebrew University - Hadassah School of Medicine, Hadassah Campus, Jerusalem, Israel

E. Pras

The Matlow's Ophthalmo-Genetics Laboratory, Department of Ophthalmology, Shamir Medical Center (Formerly Assaf-Harofeh), Tzrifin, Israel
} 
Keywords COVID-19 - SARS-CoV-2 .

Coronavirus $\cdot 2019-\mathrm{nCoV} \cdot$ Ocular

\section{Introduction}

Severe acute respiratory syndrome coronavirus 2 (SARS-CoV-2) causes the coronavirus disease 2019 (COVID-19) [1]. Since first identified in December 2019 at Wuhan, China, a global pandemic has emerged with almost 3,000,000 confirmed cases and over 200,000 deaths reported worldwide as for April 27, 2020 [2]. SARS-CoV-2 is predominantly transmitted when droplets from an infected person generate contact with mucous membranes of an uninfected individual. Currently, SARS-CoV-2 is diagnosed upon a reverse transcriptase-polymerase chain reaction (RT-PCR) assay from an oral or nasopharyngeal specimen [3].

Fever and cough are considered the hallmark of the disease. Other symptoms observed include weakness, diarrhea, sore throat and loss of smell and taste [4]. While the cumulative medical reports on the subject of COVID-19 are growing rapidly, studies examining the ocular involvement in this disease remain scarce [5-7]. Some evidence suggests that conjunctivitis could uncommonly be the first presenting symptom of COVID-19 and that ocular transmission might be an important route of transmission [8, 9]. Furthermore, previous research has described several ocular pathologies caused by other members of the coronaviruses family, including conjunctivitis, anterior and posterior uveitis, retinitis and optic nerve injury [10]. Given the close proximity between ophthalmologists and patients during examination, ophthalmologists are at increased risk of infection [11, 12].

This study aimed to evaluate the ocular manifestations in patients diagnosed with COVID-19. In addition, we aim to assess viral RNA presence from conjunctival swab samples.

\section{Methods}

This study adhered to the tenets of the Declaration of Helsinki and was approved by the institutional review board (IRB) of the Shamir Medical Center. All participants provided written informed consent prior to enrollment. No animal subjects were included in this study.

\section{Patient population}

The study group included consecutive patients admitted to the COVID-19 Ward of the Shamir Medical Center during 24/03/2020-25/04/2020 and that provided consent to participate in the study. Patients of any age were included and were required to have at least one positive SARS-CoV-2 PCR nasopharyngeal swab test.

The control group included consecutive patients admitted during a similar period to the Internal Medicine wards A, B and C of the Shamir Medical Center in a 1:2 ratio to cases. These wards admit patients who are not suspected of having COVID-19 or being carriers of SARS-CoV-2. Routine screening for patients and staff regularly occurs in these wards and all control patients were screened negative for SARSCoV-2 at RT-PCR tests.

\section{Clinical evaluation and sample acquisition}

Included patients were questioned on systemic and ocular history, current symptoms and were examined by one of two trained Ophthalmologists (IH or AS). All included patients were questioned equally on their symptoms according to a predetermined questionnaire to avoid selection bias. To reduce the risk of cross contamination, the use of a slit lamp was avoided and patients were examined with a portable set of testing aids. SARS-CoV-2 conjunctival swab samples were obtained using the standard nasopharyngeal swab, gently scraped in the inferior ocular fornix. Supplemental Figure 1 illustrates the sample acquisition technique from a patient with COVID-19 (written consent was obtained from the patient for the publication of these images). No local anesthesia or any other local treatment was instilled to the eye prior to testing in order to maintain testing accuracy. Swabs were routinely obtained from the right eye, unless apparent unilateral conjunctivitis was present, in which case the affected eye was swabbed regardless of laterality. Bacterial culture swabs were obtained in a similar manner at least $1 \mathrm{~h}$ after the viral swab was taken. These were taken in order to assess the possible effect of viral involvement on normal ocular flora. 
The main outcome measures were ocular involvement rates among patients with confirmed COVID-19, and the rate of positive viral conjunctival swab samples among patients with and without ocular involvement. Conjunctival injection was defined as symptomatic conjunctival hyperemia involving the fornices and palpebral conjunctiva. Conjunctival injection was graded on a scale of $0-4$ (0-none, 1-mild, 2-mild to moderate, 3-moderate, and 4severe). Secondary outcomes included association of ocular manifestations with blood testing results, vital signs and systemic manifestations.

COVID-19 patients were clinically categorized to mild, moderate or severe on a daily basis according to a risk assessment calculated using the Modified Early Warning Score (MEWS) [13]. Clinical variables were recorded including presenting symptoms, vital signs, laboratory results and risk assessment according to the MEWS.

\section{Laboratory procedures}

Eleven out of 16 patients with confirmed COVID-19 had ocular samples taken for SARS-CoV-2. Three patients had bacterial conjunctival swab samples taken. Samples were tested for SARS-CoV-2 using reverse-transcription polymerase chain reaction (RTPCR) analysis that target the E, N, and RdRp RNAdependent RNA polymerase genes. Oropharyngeal samples were taken as part of routine clinical care while ocular samples were taken for research purposes only. Both were tested in a clinical diagnostic laboratory at Shamir Medical Center using similar methods.

\section{Statistical analysis}

Statistical analysis was performed using IBM SPSS Statistics 25 (IBM Corp. Armonk, NY). Data are presented as mean \pm standard deviation (SD) unless otherwise specified. Categorical variables were compared using the chi-square or Fisher's Exact test, as appropriate. Clinical parameters distributions were tested for normality by the Shapiro-Wilk test. Independent and paired $T$ tests were conducted for continuous variables with a normal distribution and Wilcoxon signed-rank test and the Mann-Whitney$U$ test for variables with a non-normal distribution. $p$ values less than 0.05 on a two-sided test were considered statistically significant.

\section{Results}

Included were 48 patients, 16 with confirmed COVID19 and 32 controls who were negative for COVID-19. Baseline medical and ocular history, as well as baseline vital signs, laboratory results, symptoms and epidemiological background are detailed in Tables 1 and 2.

Active ocular involvement

Active conjunctival injection was present in three patients with COVID-19 (19\%, 3/16), all but one with bilateral presentation. Ocular discharge was present in one of the cases, as further detailed below. All three patients complained of irritation, foreign body sensation and redness. Follicular reaction was seen in one of the patients. Among the control group no patients were documented as having active conjunctival injection $(19 \%$ vs $0 \%, p=0.034)$ and none had discharge $(6 \%$ vs $0 \%, p=0.721)$. Compared with the controls, a higher percentage of patients with COVID-19 reported a foreign body sensation $(31.3 \%$ vs $3.1 \%, p=0.005)$, redness of the eye (25\% vs $0 \%, p=0.003)$, and any redness or discharge in the 30 days prior to admission (31.3\% vs $0 \%, p=0.001)$. Ocular exam findings and symptoms are further detailed in Table 3.

Among patients with COVID-19, active conjunctival injection was associated with loss of smell and loss of taste as part of the clinical presentation $(66.7 \%$ vs $7.7 \%, p=0.018$ ). A non-significant trend existed for association with a more severe disease course, as these patients had a higher MEWS at admission (median: 2 vs 1 ; mean: $3.7 \pm 3.9$ vs $2.7 \pm 1.8$, $p=0.402$ ), lower blood oxygen saturation at admission (median: $98.0 \%$ vs $99.0 \%$; mean: $95.3 \pm 5.5 \%$ vs $96.5 \pm 4.2 \%, p=0.433$ ), lower systolic blood pressure (median: $123 \mathrm{mmHg}$ vs $139 \mathrm{mmHg}$; mean: $119 \pm 6.2 \mathrm{mmHg}$ vs $139 \pm 22 \mathrm{mmHg}, p=0.193)$, higher heart rate (median: 89 BPM vs 80 BPM; mean: $96 \pm 22 \mathrm{BPM}$ vs $80 \pm 10 \mathrm{BPM}, p=0.033$ ), higher temperature (median: $37.0^{\circ} \mathrm{C}$ vs $36.7^{\circ} \mathrm{C}$; mean: $37.2 \pm 0.4{ }^{\circ} \mathrm{C}$ vs $\left.36.8 \pm 0.5^{\circ} \mathrm{C}, p=0.474\right)$ and higher white blood cell count (median: 9.0 cells per 
Table 1 Baseline patient characteristics

Baseline patient

characteristics of

consecutive patients with

confirmed COVID-19 and

patients not suspected of

having COVID-19 admitted

to Shamir medical center

during March and April

2020. Values are presented

as mean (standard

deviation) for continuous

variable and as number

(percent) for categorical

variables. COVID-19,

Coronavirus disease 2019.

COPD, chronic obstructive

pulmonary disease

${ }^{\mathrm{a}}$ In one or both eyes

\begin{tabular}{lccc}
\hline Variable & $\begin{array}{l}\text { COVID-19 patients } \\
n=16\end{array}$ & $\begin{array}{l}\text { Controls } \\
n=32\end{array}$ & $p$ value \\
\hline Age (years) & $58.7(24)$ & $65.7(19)$ & 0.286 \\
Male gender & $7(43.7)$ & $16(50.0)$ & 0.683 \\
Medical history & & & \\
Asthma & $1(6.3)$ & $1(3.1)$ & 0.610 \\
Smoker & $5(31.3)$ & $15(46.9)$ & 0.301 \\
COPD & $1(6.3)$ & $5(15.6)$ & 0.355 \\
Chronic kidney disease & $1(6.3)$ & $11(34.4)$ & 0.034 \\
Chronic dialysis & $1(6.3)$ & $2(6.3)$ & 0.746 \\
Obstructive sleep apnea & $0(0)$ & $1(3.1)$ & 0.475 \\
Stroke & $2(13.3)$ & NA & - \\
Heart failure & $2(12.5)$ & $13(12.5)$ & 0.688 \\
Diabetes mellitus & $6(37.5)$ & $21(65.6)$ & 0.147 \\
Essential hypertension & $7(43.8)$ & & \\
Ocular history & & $4(12.5)$ & 0.504 \\
Glaucoma & $1(6.3)$ & $2(6.2)$ & 0.307 \\
Dry-eye disease & $0(0)$ & $10(31)$ & 0.052 \\
Status post cataract surgery & $1(6.3)$ & $1(3.1)$ & 0.610 \\
Status post vitrectomy & $1(6.3)$ & &
\end{tabular}

$\mu \mathrm{L}$ vs 7.0 cells per $\mu \mathrm{L}$; mean: $7.6 \pm 2.3$ cells per $\mu \mathrm{L}$ vs $6.1 \pm 2.8$ cells per $\mu \mathrm{L}, p=0.327$ ).

Microbiological outcomes

Conjunctival swab testing for viral RNA were taken from 11 patients, including one of the three patients with diagnosed active conjunctival injection. All showed negative results for all three genes tested ( $\mathrm{E}$ gene, $\mathrm{N}$ gene, and RdRp gene). Bacterial conjunctival swab samples revealed growth of Staphylococcus epidermidis in one case and had no growth in two other cases.

COVID-19 patients with active conjunctival injection: case summaries

Patient \#1 was an 86-year-old female who presented with fever, cough and shortness of breath 1 week prior to her admission and was tested positive for COVID19. She was a known smoker, had asthma and ischemic heart disease. She was pseudophakic in both eyes. On admission she had low blood oxygen saturation $(90 \%)$ and was categorized as severe COVID-19 (MEWS of 9). She was started on oxygen by face mask, Hydroxychloroquine $200 \mathrm{mg}$ twice a day and received several doses of Tocilizumab. Two days after admission she complained of discharge from her left eye. On exam there was active conjunctival injection graded +3 in the left eye only with follicular reaction, associated with discharge graded +1 . She was started on antibiotic ointment with improvement. SARS-CoV-2 conjunctival swab was negative for viral RNA and bacterial culture results were positive for Staphylococcus epidermidis growth.

Patient \#2 was a 48-year-old male who presented with a 1-day history of shortness of breath and noted loss of smell and taste. He was otherwise healthy and had no ocular history. On his admission was categorized as mild (MEWS of 0 ). When actively questioned he reported irritation, redness and foreign body sensation in both eyes in the past 2 days. On exam there was active conjunctival injection graded +2 in both eyes with $\mathrm{a}+1$ watery discharge. He declined any conjunctival swabs from being taken.

Patient \#3 was a 22-year-old male who presented with a 3-day history of sore throat loss of smell and loss of taste. He was a smoker and had no ocular history. Upon admission he was categorized as mild (MEWS of 2). When actively questioned he reported on irritation and redness in both eyes for the last week. 
Table 2 Clinical and laboratory data of the study groups at presentation

\begin{tabular}{|c|c|c|c|c|}
\hline Variable & $\begin{array}{l}\text { COVID-19 patients } \\
n=16\end{array}$ & $\begin{array}{l}\text { Controls } \\
n=32\end{array}$ & Reference range & $p$ value \\
\hline \multicolumn{5}{|l|}{ Epidimiological background } \\
\hline Recent travel abroad & $1(6.2)$ & $0(0)$ & & 0.153 \\
\hline Known COVID-19 contacts & $10(63)$ & $0(0)$ & & $<0.001$ \\
\hline \multicolumn{5}{|l|}{ Symptoms } \\
\hline Cough & $11(69)$ & $3(9.4)$ & & $<0.001$ \\
\hline Shotness of breath & $7(44)$ & $11(34.4)$ & & 0.527 \\
\hline Diarrhea & $2(13)$ & $2(6.3)$ & & 0.460 \\
\hline Sore throat & $3(19)$ & $2(6.3)$ & & 0.181 \\
\hline Hyposmia & $3(19)$ & $0(0)$ & & 0.011 \\
\hline Dysgeusia & $3(19)$ & $0(0)$ & & 0.011 \\
\hline \multicolumn{5}{|l|}{ Vital signs } \\
\hline Temperature $>38.0^{\circ} \mathrm{C}$ & $9(56)$ & $5(15.6)$ & & 0.004 \\
\hline Heart rate $>100$ beats per min & $1(6)$ & $5(15.6)$ & & 0.355 \\
\hline Saturation $<96 \%$ at room air & $5(31)$ & $8(25)$ & & 0.646 \\
\hline \multicolumn{5}{|l|}{ Laboratory results } \\
\hline Hemoglobin (mg/dL) & $12.7(2.2)$ & $11.4(2.67)$ & $13.5-17.5$ & 0.113 \\
\hline White blood count (cells per $\mu \mathrm{L}$ ) & $6.1(2.3)$ & $7.4(4.7)$ & $4.0-11.0$ & 0.117 \\
\hline Neutrophiles count (cells per $\mu \mathrm{L}$ ) & $4.4(1.9)$ & $6.2(4.5)$ & $2.0-7.7$ & 0.145 \\
\hline Lymphocytes count (cells per $\mu \mathrm{L}$ ) & $1.5(0.6)$ & $1.4(0.8)$ & $1.0-4.0$ & 0.608 \\
\hline Platlets (cells per $\mu \mathrm{L}$ ) & $220(63)$ & $237(114)$ & $150-450$ & 0.298 \\
\hline Creatinine (mg/dL) & $0.82(0.22)$ & $1.04(1.03)$ & $0.7-1.20$ & 0.126 \\
\hline Alanine aminotransferase $>40$ U/liter & $0(0)$ & $9(28.1)$ & & 0.019 \\
\hline Aspartate aminotransferase $>40 \mathrm{U} /$ liter & $1(6.3)$ & $8(25)$ & & 0.117 \\
\hline Lactate dehydrogenase (mg/dL) & $460(125.8)$ & $488(360.9)$ & $240-480$ & 0.290 \\
\hline C-reactive protein $(\mathrm{mg} / \mathrm{L})$ & $18(74.6)$ & $22.9(58.8)$ & $0.3-5.00$ & 0.923 \\
\hline Pathological electrocardiogram & $2(12.5)$ & $9(33)$ & & 0.130 \\
\hline Pathological chest $X$ ray & 3 (18.8) & $13(46)$ & & 0.066 \\
\hline
\end{tabular}

Epidemiological, clinical and laboratory data of consecutive patients with confirmed COVID-19 and patients not suspected of having COVID-19 admitted to Shamir medical center during March and April 2020. Values are presented as mean (standard deviation) for continuous variable and as number (percent) for categorical variables. COVID-19, Coronavirus disease 2019

On exam there was active conjunctival injection graded +2 in both eyes with no discharge. He declined any conjunctival swabs from being taken.

\section{Discussion}

In this study, we evaluated the ocular signs and symptoms, as well as the presence of SARS-CoV-2 in conjunctival swab samples among patients with COVID-19 in one tertiary referral center during March and April of 2020. Outcomes were compared to a control group of patients negative for COVID-19, admitted during a similar period to a different ward. The results show that active conjunctival injection was present in 19\% of cases and was associated with loss of smell and taste as part of the clinical presentation. Patients with COVID-19 were also more likely to complain of foreign body sensation and redness of the eye compared to patients negative for COVID-19. Conjunctival swabs for viral RNA were negative in patients with and without ocular involvement.

To date, little is known regarding the presence of SARS-CoV-2 on the ocular surface. Currently there 
Table 3 Ocular signs and symptoms

\begin{tabular}{|c|c|c|c|}
\hline Variable & $\begin{array}{l}\text { COVID-19 patients } \\
n=16\end{array}$ & $\begin{array}{l}\text { Controls } \\
n=32\end{array}$ & $p$ value \\
\hline \multicolumn{4}{|l|}{ Ocular symptoms } \\
\hline Irritation $^{\mathrm{a}}$ & $5(31.3)$ & $4(12.5)$ & 0.121 \\
\hline Foreign body sensation $^{a}$ & $5(31.3)$ & $1(3.1)$ & 0.005 \\
\hline Discharge $^{\mathrm{a}}$ & $1(6.3)$ & $0(0)$ & 0.153 \\
\hline Red eye $^{\mathrm{a}}$ & $4(25)$ & $0(0)$ & 0.003 \\
\hline Prior conjunctivitis ${ }^{\mathrm{b}}$ & $5(31.3)$ & $0(0)$ & 0.001 \\
\hline \multicolumn{4}{|l|}{ Ocular examination findings } \\
\hline Conjunctival injection $\geq+2^{\mathrm{c}}$ & $3(19)$ & $0(0)$ & 0.034 \\
\hline Discharge $\geq+1^{\mathrm{c}}$ & $1(6.2)$ & $0(0)$ & 0.153 \\
\hline Eyelid erythema $\geq+1^{c}$ & $1(6.2)$ & $0(0)$ & 0.153 \\
\hline
\end{tabular}

Ocular signs and symptoms of consecutive patients with confirmed COVID-19 and patients not suspected of having COVID-19 admitted to Shamir medical center during March and April 2020. Values are presented as mean (standard deviation) for continuous variable and as number (percent) for categorical variables. COVID-19, Coronavirus disease 2019

${ }^{\mathrm{a}}$ As reported by the patient on a scale of $0-10$

${ }^{\mathrm{b}}$ Any redness or discharge in the 30 days prior to admission

${ }^{\mathrm{c}}$ As assessed by the examining ophthalmologist between 0 and 4

are limited studies that report on the subject, all from East Asia [5, 7, 14, 15]. Reported positive conjunctival swab rates were between 0 and 5\%. Furthermore, a recent meta-analysis reported that the pooled sensitivity of ocular tissue and fluid in detecting SARSCoV-2 was only $0.6 \%$ in comparison with nasopharyngeal and sputum swabs [16]. It is interesting to note the recent publication by Seah et al. who examined 64 samples from 17 patients using a Schirmer test strip. They reported all examinations negative for viral RNA [17]. Our sampling technique was different. While Shea et al. focused on the existence of viral load in the tears, the method we employed benefits from direct scraping of conjunctival cells. The similarity in results however is consistent with the presumption of a low viral load in this tissue.

In our study none of the conjunctival swab samples were positive for SARS-CoV-2. This result could have been influenced by the limited sensitivity of the test, which was not designed or validated for conjunctival sampling, but rather for oropharyngeal testing. Another interesting possibility however relates to the limited viral load present in the conjunctiva. This possibility could have implications on the infectious capacity of this tissue. Some evidence suggests that droplets expelled from the mouth or nose of a patient can come in contact with an examiner's eyes, forming an avenue of infection [8]. However the authors could find no evidence of infection originating from conjunctival tissue of an infected patient. Which appears in agreement with our results here. This could be an encouraging fact for ophthalmologists, who should nevertheless be highly protected due to the close proximity to patients during examination.

Active conjunctival involvement was associated with loss of smell and taste as part of the clinical presentation and a trend existed for a more severe disease course. Loss of smell or taste was a relatively late symptom to be revealed as associated with the disease. Little is currently known on the pathophysiologic mechanism by which it develops $[18,19]$. We theorize that ocular involvement was associated with increased viral load in the nasal cavity and could perhaps have led to this association.

Previous studies have also suggested that ocular involvement is associated with a more severe disease presentation [5]. Several clinical measures related to disease severity were more pronounced among patients with ocular involvement in our study group. However, these did not reach statistical significance. The inability to reach statistical significance might be related to our exclusion of highly severe cases on 
account of their inability to provide consent to participate in the study. In addition, the relatively young patient age seen in our patient group (59 \pm 24 years), might also have inadvertently excluded more severe cases. Patient's mean age might have been influenced by the lesser ability of older patients to provide consent given the more severe manifestations of the disease at older ages. More data is needed, however conjunctival involvement should prompt the treating physicians to carefully monitor and evaluate the patient for other markers of disease severity.

It is interesting to note that ocular involvement was seen as a late presentation in patient \#1, with ocular symptoms appearing at 9 days (after the onset of respiratory symptoms and 2 days into her admission), while in patients \#2 and \#3 ocular symptoms preceded respiratory ones. In patient \#2 they occurred 1 day prior to respiratory symptoms onset (which coincided with loss of smell and taste) and in patient \#3, 4 days prior to respiratory symptoms onset (sore throat and loss of smell and of taste). It should also be noted that when COVID-19 patients were questioned on redness or discharge in the 30 days prior to admission, five (Patients \#2, \#3 and three additional patients) responded positively (compared to none in the control group, $p=0.001$, Table 3). A review of their charts reveals that the three additional patients had respiratory symptoms well before admission to our medical facility. This might suggest that these three patients with COVID-19 could have had ocular involvement at early stages as well. However due to the fact that these resolved, and could not be verified in clinical examination, they were excluded from the primary analysis.

In April of 2020 Neri and Pichi [20] suggested that an animal model where murine coronavirus induced an acute and long-lasting disease of the retina might serve as a model to examine COVID-19 associated inflammatory reaction. This model termed experimental coronavirus retinopathy (ECOR), suggests that SARS-COV-2 could have tropism for ocular tissues and induce a primary infection triggering the immune system, followed by a second autoimmune stage in which postviral inflammation can develop. Although in the ECOR model, using murine coronavirus, specific retinotropism was seen, humans affected by SARS-COV-2 might react differently, in ways also affecting the ocular surface. It would be interesting to follow the long-term retinal involvement of patients affected by COVID-19-associated conjunctivitis.

This study has several limitations. First, to reduce the risk of cross contamination of equipment, the use of a slit-lamp was avoided, as was indirect ophthalmoscopy. We therefore cannot comment on the rates of anterior or posterior uveitis as well as retinal pathologies. Second, patients with a highly severe course of disease were inherently excluded as they were unable to provide consent. Third, ocular sampling for SARS-CoV-2 among patients with active conjunctival injection were available in only one patient. Finally, this study included a relatively small sample size. The strengths of this study include the combination of clinical and molecular analysis and the use of an independent control group, as well as the assessment of a patient population outside of East Asia.

To conclude, among patients admitted to a tertiary referral center with confirmed COVID-19, active conjunctival injection was present in $19 \%$ of cases and was associated with loss of smell and taste as part of the clinical presentation. Patients with COVID-19 were also more likely to complain of foreign body sensation and redness of the eye compared to those negative for COVID-19. Conjunctival swabs for viral RNA were negative in patients with and without ocular involvement. These results could have implications on screening and triage of patients affected by COVID-19 as well as on implementation of appropriate protective measures for ophthalmology staff treating affected patients.

Funding None.

\section{Compliance with ethical standards}

Conflict of interest All authors declare that they have no conflict of interest.

Ethical approval All procedures performed in studies involving human participants were in accordance with the ethical standards of the institutional research committee and with the 1964 Helsinki declaration and its later amendments or comparable ethical standards.

Informed consent Informed consent was obtained from all individual participants included in the study. 


\section{References}

1. Li Q, Guan X, Wu P et al (2020) Early transmission dynamics in Wuhan, China, of novel coronavirus-infected pneumonia. N Engl J Med 382(13):1199-1207. https://doi. org/10.1056/NEJMoa2001316

2. Worldometer (2020) COVID-19 coronavirus pandemic. Worldometer. https://doi.org/10.1101/2020.01.23. 20018549V2

3. World Health Organization: Coronavirus disease (COVID19) technical guidance: Laboratory testing for 2019-nCoV in humans. Accessed 24 April 2020. https://doi.org/10. 1080/00963402.1970.11457861

4. Guan W, Ni Z, Hu Y et al (2020) Clinical characteristics of coronavirus disease 2019 in China. N Engl J Med. https:// doi.org/10.1056/nejmoa2002032

5. Wu P, Duan F, Luo C et al (2019) Characteristics of ocular findings of patients with coronavirus disease 2019 (COVID19) in Hubei Province, China. JAMA Ophthalmol. https:// doi.org/10.1001/jamaophthalmol.2020.1291

6. Yu A-Y, Tu R, Shao X, Pan A, Zhou K, Huang J (2020) A comprehensive Chinese experience against SARS-CoV-2 in ophthalmology. Eye Vis 7(1):19. https://doi.org/10.1186/ s40662-020-00187-2

7. Zhang X, Chen X, Chen L et al (2020) The evidence of SARS-CoV-2 infection on ocular surface. Ocul Surf. https:// doi.org/10.1016/j.jtos.2020.03.010

8. Wei LC, Fen LX, Fang JZ (2020) 2019-nCoV transmission through the ocular surface must not be ignored. Lancet 395(10224):e39. https://doi.org/10.1016/S01406736(20)30313-5

9. Zhou Y, Zeng Y, Tong Y, Chen C (2020) Ophthalmologic evidence against the interpersonal transmission of 2019 novel coronavirus through conjunctiva. medRxiv. https:// doi.org/10.1101/2020.02.11.20021956

10. Seah I, Agrawal R (2020) Can the coronavirus disease 2019 (COVID-19) affect the eyes? A review of coronaviruses and ocular implications in humans and animals. Ocul Immunol Inflamm 28(3):391-395. https://doi.org/10.1080/09273948. 2020.1738501

11. Patel A, Jernigan DB, Abdirizak F et al (2020) Initial public health response and interim clinical guidance for the 2019 novel coronavirus outbreak-United States, December 31,
2019-February 4, 2020. Am J Transplant 20(3):889-895. https://doi.org/10.1111/ajt.15805

12. Lai THT, Tang EWH, Chau SKY, Fung KSC, Li KKW (2020) Stepping up infection control measures in ophthalmology during the novel coronavirus outbreak: an experience from Hong Kong. Graefe's Arch Clin Exp Ophthalmol. https://doi.org/10.1007/s00417-020-04641-8

13. Song C-Y, Xu J, He J-Q, Lu Y-Q (2020) COVID-19 early warning score: a multi-parameter screening tool to identify highly suspected patients. medRxiv. https://doi.org/10. 1101/2020.03.05.20031906

14. Chen L, Liu M, Zhang Z et al (2020) Ocular manifestations of a hospitalised patient with confirmed 2019 novel coronavirus disease. Br J Ophthalmol. https://doi.org/10.1136/ bjophthalmol-2020-316304

15. Xia J, Tong J, Liu M, Shen Y, Guo D (2020) Evaluation of coronavirus in tears and conjunctival secretions of patients with SARS-CoV-2 infection. J Med Virol. https://doi.org/ 10.1002/jmv. 25725

16. Ulhaq ZS, Soraya GV (2020) The prevalence of ophthalmic manifestations in COVID-19 and the diagnostic value of ocular tissue/fluid. Graefe's Arch Clin Exp Ophthalmol. https://doi.org/10.1007/s00417-020-04695-8

17. Yu Jun IS, Anderson DE, Zheng Kang AE et al (2020) Assessing viral shedding and infectivity of tears in coronavirus disease 2019 (COVID-19) patients. Ophthalmology. https://doi.org/10.1016/j.ophtha.2020.03.026

18. Gautier JF, Ravussin Y (2020) A new symptom of COVID19: loss of taste and smell. Obesity. https://doi.org/10.1002/ oby. 22809

19. Vaira LA, Salzano G, Deiana G, De Riu G (2020) Anosmia and ageusia: common findings in COVID-19 patients. Laryngoscope. https://doi.org/10.1002/lary.28692

20. Neri P, Pichi F (2020) COVID-19 and the eye immunity: lesson learned from the past and possible new therapeutic insights. Int Ophthalmol 40(5):1057-1060. https://doi.org/ 10.1007/s10792-020-01389-2

Publisher's Note Springer Nature remains neutral with regard to jurisdictional claims in published maps and institutional affiliations. 